\title{
POLYNOMIAL DECAY FOR SOLUTIONS OF HYPERBOLIC INTEGRODIFFERENTIAL EQUATIONS*
}

\author{
T. BÁRTA \\ Department of Mathematical Analysis, Faculty of Mathematics and Physics, Charles University, Prague, \\ Sokolovska 83, 18000 Prague 8, Czech Republic \\ e-mail: barta@karlin.mff.cuni.cz
}

(Received 9 October 2007; accepted 22 May 2008)

\begin{abstract}
We consider a linear integrodifferential equation of second order in a Hilbert space and show that the solution tends to zero polynomially if the decay of the convolution kernel is polynomial. Both polynomials are of the same order.
\end{abstract}

2000 Mathematics Subject Classification. 45D05.

1. Introduction. In this paper, we investigate the following integrodifferential equation

$$
\begin{aligned}
& \ddot{u}(t)=-A u(t)+\int_{0}^{t} g(t-s) A u(s) d s+f(t), \\
& u(0)=x, \quad \dot{u}(0)=y
\end{aligned}
$$

in a Hilbert space $H$. We generalize the result by Rivera and Gomez [1] on polynomial decay of the solutions. As in [1], $A: D(A) \rightarrow H$ is a self-adjoint operator. Our assumptions on $g$ are as follows:

(g1) $g(t) \in C^{3}([0,+\infty)), g(t)>0$ for all $t \geq 0$.

(g2) There exists $c_{0}>0$ such that $-c_{0} g(t) \leq g^{\prime}(t)$ for all $t \geq 0$.

(g3) There exist $c_{1}>0$ and $p>1$ such that $g^{\prime}(t) \leq-c_{1} g^{1+\frac{1}{p}}(t)$ for all $t \geq 0$.

(g4) There exists $c_{2}>0$ such that $\left|g^{\prime \prime}(t)\right| \leq c_{2} g(t)$ for all $t \geq 0$.

(g5) $G:=\int_{0}^{\infty} g(\tau) d \tau<1$.

It follows from (g3) and continuity of $g$ in 0 that $g(t) \leq C(1+t)^{-p}$ for some $C>0$. Unlike Rivera and Gomez, we do not need $p>2$ and our assumption (g2) is also weak. In fact, in [1] one assumes $-c_{0} g(t)^{1+\frac{1}{p}} \leq g^{\prime}(t)$ which means that the behaviour of $g$ in $+\infty$ is exactly the same as $t^{-p}$. This excludes kernels like $g(s)=(1+t)^{-p} \ln (1+t)$. In our case, the decay of $g$ is anything between polynomial and exponential.

Throughout this paper, $c$ and $C$ are general positive constants independent of $t$; their values vary from expression to expression.

2. Main result. We introduce an energy functional and formulate the main result. Define

$$
E(t, v):=\frac{1}{2}\left(\left\|v_{t}\right\|^{2}+(1-G(t))\left\|A^{1 / 2} v\right\|^{2}+g \circ A^{1 / 2} v\right)
$$

$\overline{{ }^{*} \text { This work }}$ is a part of the project MSM 0021620839 and was partly supported by GACR 201/06/P171. 
where

$$
(g \circ k)(t):=\int_{0}^{t} g(t-s)\|k(s)-k(t)\|^{2} d s
$$

and

$$
G(t):=\int_{0}^{t} g(s) d s .
$$

THEOREM 2.1. Let $g$ satisfy $(g 1)-(g 5)$ and $A$ be a self-adjoint operator (such that $D\left(A^{r}\right) \hookrightarrow D\left(A^{s}\right)$ is compact for $\left.r>s\right)$. Let $x \in D(A), y \in D\left(A^{1 / 2}\right)$ and $f \in C^{1}\left(\mathbb{R}_{+}, H\right)$ such that

$$
\|f(t)\|^{2} \leq \frac{c_{f}}{(1+t)^{p}}
$$

for a positive constant $c_{f}$. Then there exists $C_{E}>0$ such that the solution $u$ of (1) satisfies

$$
E(t, u) \leq C_{E} E(0, u) \frac{1}{(1+t)^{p}} .
$$

First of all, according to [2], there exists a global solution $u \in C^{2}\left(\mathbb{R}_{+}, H\right) \cap$ $C^{1}\left(\mathbb{R}_{+}, D\left(A^{1 / 2}\right)\right) \cap C\left(\mathbb{R}_{+}, D(A)\right)$ of $(1)$ whenever $x \in D(A), y \in D\left(A^{1 / 2}\right)$. From now on, $u$ is the solution of (1). Let us start proving Theorem 2.1. The following lemmas will be helpful.

\section{LEMMA 2.2. Denote}

$$
w(t):=u(t)-(g * u)(t) .
$$

Then there exist $K, k>0$, such that the following estimates hold for all $t \in \mathbb{R}_{+}$(the values of $k$ and $K$ in different lines may be different).

$$
\begin{aligned}
\|w\|^{2} & \leq K\left(\|u\|^{2}+g \circ u\right), \\
\left\|w_{t}\right\|^{2} & \leq K\left(\left\|u_{t}\right\|^{2}+g(t)\|u\|^{2}+g \circ u\right), \\
\left\|A^{1 / 2} w\right\|^{2} & \leq K\left(\left\|A^{1 / 2} u\right\|^{2}+g \circ A^{1 / 2} u\right), \\
\left\|w_{t}\right\|^{2} & \geq k\left\|u_{t}\right\|^{2}-K\left(g(t)\|u\|^{2}+g \circ u\right), \\
\left\|A^{1 / 2} w\right\|^{2} & \geq k\left((1-G(t))\left\|A^{1 / 2} u\right\|^{2}\right)-K g \circ A^{1 / 2} u .
\end{aligned}
$$

Proof. To prove the first estimate we multiply (3) by $w$

$$
\|w(t)\|^{2}=(u(t), w(t))-((g * u)(t), w(t)) .
$$

For every $c>0$ there exists $C>0$ such that

$$
(u, w) \leq C\|u\|^{2}+c\|w\|^{2}
$$

and

$$
\begin{aligned}
\left|\int_{0}^{t} g(t-s)(u(s), w(t)) d s\right| \leq & \left|\int_{0}^{t} g(t-s)(u(s)-u(t), w(t)) d s\right| \\
& +\left|\int_{0}^{t} g(t-s)(u(t), w(t)) d s\right|
\end{aligned}
$$




$$
\begin{aligned}
\leq & \int_{0}^{t} g(t-s)\left(C\|u(s)-u(t)\|^{2}+c\|w(t)\|^{2}\right) d s \\
& +\int_{0}^{t} g(t-s)\left(C\|u(t)\|^{2}+c\|w(t)\|^{2}\right) d s \\
\leq & C(g \circ u)(t)+c G(t)\|w(t)\|^{2}+C G(t)\|u(t)\|^{2}+c G(t)\|w(t)\|^{2} .
\end{aligned}
$$

Inserting these estimates into (4) we obtain $(G(t)<1$ by (g5))

$$
(1-3 c)\|w(t)\|^{2} \leq 2 C\|u(t)\|^{2}+C(g \circ u)(t) .
$$

Taking $c$ small enough we have proved the first estimate with $K:=2 C /(1-3 c)$.

To show the second estimate, we multiply the derivative of (3) by $w_{t}$

$$
\left\|w_{t}(t)\right\|^{2}=\left(u_{t}(t), w_{t}(t)\right)-\left(\partial_{t}(g * u)(t), w_{t}(t)\right) .
$$

The first term on the right-hand side is estimated as in (5) and the second term can be rewritten as

$$
\begin{gathered}
g(0)\left(u(t), w_{t}(t)\right)+\int_{0}^{t} g^{\prime}(t-s)\left(u(s)-u(t), w_{t}(t)\right) d s+\int_{0}^{t} g^{\prime}(t-s)\left(u(t), w_{t}(t)\right) d s \\
=g(t)\left(u(t), w_{t}(t)\right)+\int_{0}^{t} g^{\prime}(t-s)\left(u(s)-u(t), w_{t}(t)\right) d s .
\end{gathered}
$$

According to (g2), the integral term can be estimated by

$$
c_{0}\left(C(g \circ u)(t)+c G(t)\|w(t)\|^{2}\right) .
$$

Inserting the estimates into (6) we obtain

$$
\left(1-c-g(t) c-c_{0} c\right)\left\|w_{t}\right\|^{2} \leq C\left\|u_{t}(t)\right\|^{2}+C g(t)\|u(t)\|^{2}+c_{0} C g \circ u(t),
$$

and taking $c$ small enough we obtain the second estimate with $K:=C\left(1+c_{0}\right) /(1-$ $\left.c-c g(0)-c c_{0}\right)(g$ is decreasing $)$.

By the same technique we obtain the other three estimates. The third estimate follows by applying $A$ to (3) and multiplying by $w$. To show the fourth and fifth estimates we differentiate (3), resp. apply $A$ to (3), and then multiply by $u_{t}$, resp. $u$. In this proof we have applied assumptions (g1), (g2) and (g5).

It is not important in Lemma 2.2 that $u$ is the solution of (1). In fact, the estimates hold for all $u \in C\left(\mathbb{R}_{+}, D(A)\right) \cap C^{1}\left(\mathbb{R}_{+}, D\left(A^{1 / 2}\right)\right)$ and the constants are independent of $u$.

Define $\tilde{E}$ by

$$
\tilde{E}(t):=\left(\left\|w_{t}\right\|^{2}+\left\|A^{1 / 2} w\right\|^{2}+g(0)\left(w, w_{t}\right)+\frac{g(0)}{2}\|w\|^{2}\right) .
$$

It follows from Lemma 2.2, Cauchy-Schwarz inequality and $\|u\| \leq\left\|A^{1 / 2} u\right\|$ that

$$
\begin{aligned}
& c\left(\left\|u_{t}\right\|^{2}+\left\|A^{1 / 2} u\right\|^{2}\right)-C\left(g \circ A^{1 / 2} u+\|u\|^{2}+g \circ u\right) \\
& \quad \leq \tilde{E}(t) \leq C\left(\left\|u_{t}\right\|^{2}+\left\|A^{1 / 2} u\right\|^{2}+g \circ A^{1 / 2} u\right)
\end{aligned}
$$


for some $c, C>0$. Moreover, the derivatives of $E(t, u)$ and $\tilde{E}(t)$ satisfy the following estimates.

\section{LEMMA 2.3. It holds that}

$$
\frac{d}{d t} E(t, u)=-\frac{1}{2} g(t)\left\|A^{1 / 2} u\right\|^{2}+\frac{1}{2} g^{\prime} \circ A^{1 / 2} u+\left(f, u_{t}\right)
$$

and for every $\delta>0$ small enough there exists $C_{\delta}>0$ such that

$$
\begin{aligned}
\frac{d}{d t} \tilde{E}(t, u) \leq & -\left(\frac{g(0)}{2}-\delta\right)\left(\left\|w_{t}\right\|^{2}+\left\|A^{1 / 2} w\right\|^{2}\right) \\
& +C_{\delta}\left(g(t)\|u\|^{2}+g \circ u\right)+\left(f, w_{t}+\frac{g(0)}{2} w\right) .
\end{aligned}
$$

Both parts of this lemma are proved in [1]. The equality (8) follows from multiplicating (1) by $u_{t}$ and some computation; the inequality (9) can be proved in the same way as Lemma 3.2 in [1]. Assumptions (g1), (g2), (g4) and (g5) are applied.

Lemma 2.4. Let $p>1$ and $q \geq 0$. Assume that $g(t) \leq C_{1}(1+t)^{-p}$ and $\left\|k^{2}(t)\right\| \leq$ $C_{2}(1+t)^{-q}$ for some $C_{1}, C_{2}>0$ and all $t \geq 0$. If $0 \leq q \leq 1$, then for every $1>r>$ $(1-q) / p$ there exists $K>0$ such that

$$
g \circ k \leq K\left(g^{1+\frac{1}{p}} \circ k\right)^{\frac{(1-r) p}{1+(1-r) p}} \quad \text { for all } t \geq 0 .
$$

If $q>1$, then there exists $K>0$ such that

$$
g \circ k \leq K\left(g^{1+\frac{1}{p}} \circ k\right)^{\frac{p}{1+p}} \quad \text { for all } t \geq 0 .
$$

Proof. By Hölder inequality we have for $1<a<+\infty$

$$
\begin{aligned}
(g \circ k)(t)= & \int_{0}^{t} g^{\frac{1+\frac{1}{p}}{a}}(t-s)\|k(s)-k(t)\|^{\frac{1}{a}} g(t-s)^{1-\frac{1+\frac{1}{p}}{a}}(t-s)\|k(s)-k(t)\|^{1-\frac{1}{a}} d s \\
\leq & \left(\int_{0}^{t}\left(g^{\frac{1+\frac{1}{p}}{a}}(t-s)\|k(s)-k(t)\|^{\frac{2}{a}}\right)^{a} d s\right)^{\frac{1}{a}} \\
& \times\left(\int_{0}^{t}\left(g^{1-\frac{1+\frac{1}{p}}{a}}(t-s)\|k(s)-k(t)\|^{2-\frac{2}{a}}\right)^{\frac{a}{a-1}} d s\right)^{1-\frac{1}{a}} \\
= & \left(\int_{0}^{t} g^{1+\frac{1}{p}}(t-s)\|k(s)-k(t)\|^{2}\right)^{\frac{1}{a}}\left(\int_{0}^{t} g^{\frac{a-1-\frac{1}{p}}{a-1}}(t-s)\|k(s)-k(t)\|^{2} d s\right)^{1-\frac{1}{a}} .
\end{aligned}
$$

Here the first integral on the right-hand side is exactly $g^{1+\frac{1}{p}} \circ k$, so it remains to show that the second integral is bounded by a constant independent of $t$ for an appropriate $a$.

Denote

$$
r:=\frac{a-1-\frac{1}{p}}{a-1} .
$$


Then $r \in(-\infty, 1)$. Since $\|k(s)-k(t)\|^{2} \leq 2\left(\|k(s)\|^{2}+\|k(t)\|^{2}\right)$, we can split the last integral in (10) into sum of two terms.

$$
2 \int_{0}^{t} g^{r}(t-s)\|k(s)\|^{2} d s+2 \int_{0}^{t} g^{r} a-1(t-s)\|k(t)\|^{2} d s .
$$

Let $0<q \leq 1$. Then the first term in (11) is estimated as follows.

$$
\begin{aligned}
& \int_{0}^{t} g^{r}(t-s)\|k(s)\|^{2} d s \\
& \quad \leq C_{1}^{r} C_{2} \int_{0}^{t}(1+t-s)^{-p r}(1+s)^{-q} d s \\
& \quad \leq C_{1}^{r} C_{2}\left(\int_{0}^{t}\left((1+s)^{-q}\right)^{\frac{1+\varepsilon}{q}} d s\right)^{\frac{q}{1+\varepsilon}}\left(\int_{0}^{t}\left((1+t-s)^{-p r}\right)^{\frac{1+\varepsilon}{1+\varepsilon-q}} d s\right)^{1-\frac{q}{1+\varepsilon}} \leq C,
\end{aligned}
$$

provided

$$
p r \frac{1+\varepsilon}{1+\varepsilon-q}>1 \text {, i.e., } r>\frac{1-q}{p}
$$

since $\varepsilon>0$ is arbitrary. For the second term in (11), it holds

$$
\int_{0}^{t} g^{r}(t-s)\|k(t)\|^{2} d s \leq C_{1} C_{2}(1+t)^{-q} \int_{0}^{t}(1+t-s)^{-p r} d s \leq C(1+t)^{-q-p r+1} .
$$

This is bounded if

$$
1-q-p r \leq 0, \text { i.e., } r \geq \frac{1-q}{p} .
$$

Hence, if $1>r>(1-q) / p$ we have

$$
g \circ k \leq K\left(g^{1+\frac{1}{p}} \circ k\right)^{\frac{1}{a}}=K\left(g^{1+\frac{1}{p}} \circ k\right)^{\frac{(1-r) p}{1+(1-r) p}} .
$$

If $q=0$, then the first term in (11) can be estimated in the same way as the second term. If $q>1$, then the second integral in (10) is estimated by

$$
2 g(0)\left(\int_{0}^{t}\|k(s)\|^{2} d s+t\|k(t)\|^{2}\right) \leq 2 g(0) \tilde{C}(1+t)^{1-q} \leq K,
$$

provided $a \geq 1+\frac{1}{p}$. The assertion for $q>1$ follows.

LEMMA 2.5. Let $p>1$ and $k>0$ such that $\|f(t)\|^{2} \leq k(1+t)^{-p-1}$ and $g \leq k(1+$ $t)^{-p}$. Let $1 \geq q \geq 0$ such that $\left\|A^{1 / 2} u(t)\right\|^{2} \leq k(1+t)^{-q}$. Then $\left\|A^{1 / 2} u(t)\right\| \leq K(1+t)^{-\tilde{q}}$ for some $K>0$ and $\tilde{q}=q+\varepsilon$, where $\varepsilon>0$ is small enough, depending on put independent of $q$.

Proof. By the previous Lemma we have

$$
g \circ A^{1 / 2} u(t) \leq C\left(g^{1+\frac{1}{p}} \circ A^{1 / 2} u(t)\right)^{\frac{(1-r) p}{1+(1-r) p}}
$$

for all $1>r>(1-q) / p$. Take $L(t):=v E(t, u)+\tilde{E}(t)$ for $v>0$ large enough. The following estimate follows from Lemma 2.3 by applying Cauchy-Schwarz inequality 
to the terms containing $f$, assumption (g3) to the term containing $g^{\prime}$ and Lemma 2.2 to the terms containing $w$.

$$
\begin{aligned}
\frac{d}{d t} L(t) \leq & v\left(-\frac{1}{2} g(t)\left\|A^{1 / 2} u(t)\right\|^{2}-\frac{1}{2} g^{1+\frac{1}{p}} \circ A^{1 / 2} u(t)\right)-C\left((1-G(t))\left\|A^{1 / 2} u(t)\right\|^{2} .\right. \\
& \left.+(1-\delta)\left\|u_{t}\right\|^{2}\right)+g(t)\|u(t)\|^{2}+C_{\delta}\left(g \circ u+g \circ A^{1 / 2} u\right)+v C_{\delta}\|f\|^{2} .
\end{aligned}
$$

Here $0<\delta<1$ and $C, C_{\delta}>0$. By $\|u(t)\| \leq c\left\|A^{1 / 2} u(t)\right\|$ and (12) we obtain for $v$ large enough

$$
\begin{aligned}
\frac{d}{d t} L(t) & \leq-C\left((1-G(t))\left\|A^{1 / 2} u(t)\right\|^{2}+\left\|u_{t}\right\|^{2}\right)-C\left(g \circ A^{1 / 2} u(t)\right)^{\frac{1+(1-r) p}{(1-r) p}}+v C_{\delta}\|f\|^{2} \\
& \leq-C\left((1-G(t))\left\|A^{1 / 2} u(t)\right\|^{2}+\left\|u_{t}\right\|^{2}+g \circ A^{1 / 2} u(t)\right)^{\frac{1+(1-r) p}{(1-r) p}}+v C_{\delta}\|f\|^{2} .
\end{aligned}
$$

Since $\tilde{E}(t) \leq c E(t, u)$, we obtain

$$
\frac{d}{d t} L(t) \leq-C(L(t))^{\frac{1+(1-r) p}{(1-r) p}}+v C_{\delta}\|f\|^{2} .
$$

Hence,

$$
L(t) \leq C L(0)(1+t)^{(1-r) p} \quad \text { and also } \quad\left\|A^{1 / 2} u(t)\right\|^{2} \leq C L(0)(1+t)^{(1-r) p} .
$$

Let $0<\tilde{\varepsilon}<1-1 / p$. Set $r:=(1-q) / p+\tilde{\varepsilon}$ and $\tilde{q}:=(1-r) p$. Then $1>r>(1-q) / p$ and $\tilde{q}=q+(p-1-\tilde{\varepsilon} p)>q$. We have proved the assertion with $\tilde{q}=q+\varepsilon$, where $\varepsilon=p-1-\tilde{\varepsilon} p>0$ is independent of $q$.

Lemma 2.6. There exists $C>0$ such that $\left\|A^{1 / 2} u(t)\right\| \leq C$ for all $t \geq 0$.

Proof. According to Theorem 5.1 in [2], the solution $v$ of the homogeneous equation

$$
\begin{aligned}
& \ddot{u}(t)=-A u(t)-\int_{0}^{t} g(t-s) A u(s) d s, \\
& u(0)=x, \quad \dot{u}(0)=y,
\end{aligned}
$$

satisfies $v, \dot{v} \in L^{2}\left(\mathbb{R}_{+}, X\right)$. Integrating (1) we obtain

$$
\dot{u}(t)=-\int_{0}^{t} \tilde{G}(t-s) A u(s) d s+F(t)+y,
$$

where $\tilde{G}(\cdot)$ is the primitive function of $g$ with $G(0)=1$ and $F(t):=\int_{0}^{t} f(s) d s$. It follows that the solution of the inhomogeneous equation is given by

$$
u(t):=v(t)+\int_{0}^{t}(F(t-s)+y) v(s) d s .
$$

Hence,

$$
\dot{u}(t)=\dot{v}(t)+(F(0)+y) v(t)+\int_{0}^{t} f(t-s) v(s) .
$$


Since $v, \dot{v} \in L^{2}$ and $f \in L^{1}$, we obtain that $\dot{u} \in L^{2}$. Now it follows from (8) that

$$
E(t, u) \leq E(0, u)+\int_{0}^{t}\|f(s)\| \cdot\|\dot{u}(s)\| d s \leq E(0, u)+\|f\|_{2}\|\dot{u}\|_{2} .
$$

Hence, $\left\|A^{1 / 2} u\right\|^{2}$ is bounded.

We will finish the proof of Theorem 2.1. Since $\left\|A^{1 / 2} u(t)\right\|$ is bounded, i.e., assumptions of Lemma 2.5 hold, we obtain

$$
\left\|A^{1 / 2} u(t)\right\| \leq c(1+t)^{-q}
$$

for some $q>1$ by applying Lemma 2.5 finitely many times. Then by Lemma 2.4 we obtain (12) with $r=0$ and the proof of Lemma 2.5 yields (see (13))

$$
L(t) \leq C L(0)(1+t)^{p} .
$$

Estimating $\tilde{E}$ according to (7), we obtain $v E(t, u)+\tilde{E}(t) \geq(v-C) E(t, u)$. Hence, (2) holds.

\section{REFERENCES}

1. J. E. M. Rivera and F. P. Q. Gomez, Existence and decay in non-linear viscoelasticity, Boll.U.M.I. 6-B (8) (2003) 1-37.

2. R. K. Miller and R. L. Wheeler, Well-posedness and stability of linear Volterra integrodifferential equations in abstract spaces, Funkcialaj Ekvacioj 21 (1978) 279-305. 